Obwohl Brownlies ,Principles“ eine Reihe durchaus prinzipieller Fragen unbeantwortet lassen, wird man auf dieses Buch, dessen Vorrangstellung in der angelsächsischen Völkerrechtsdoktrin weithin gerechtfertigt erscheint, immer wieder zurückzugreifen haben.

Alexander Dix

Alan J. Day/Henry W. Degenhardt (comp. and ed.)

\title{
Political Parties of the World
}

A Kessing's Reference Publication, Longman, Essex, 1980, 432 S.

Diese Zusammenstellung von Mitarbeitern der Keesing's Contemporary Archives kann als nützlicher Einstieg in die komparative Parteienforschung genutzt werden. Sie bietet erste Hinweise und meist auch die Adressen der aufgeführten Parteien, mit denen weitergearbeitet werden kann. Den Autoren geht es um Vollständigkeit sowohl was die Länder und Territorien ( 211 werden aufgeführt) als auch was die Parteienbiographien betrifft (54 werden allein für die Bundesrepublik aufgeführt). Die einzelnen Beiträge selbst sind sehr knapp gehalten (zwischen 10 Zeilen und einer guten halben Seite). Es wird das Gründungsdatum, der gegenwärtige Parteiführer, die Geschichte, Zielsetzung, gelegentlich die Struktur und Bedeutung (bei Wahlen, Parteimitgliedschaft), Publikationsorgane sowie für jedes Land die Sitzverteilung im zuletzt gewählten Parlament erwähnt. Die Parteinamen werden sowohl in ihrer Ursprungsbezeichnung als auch in englischer Ubersetzung aufgeführt. Im Anhang werden die Mitgliederparteien der verschiedenen Partienweltbünde sowie die Sitze der Parteiengruppierungen im Europäischen Parlament genannt.

Rolf Hanisch

Jens Muller

Liquidation or Consolidation of Indigenous Technology

A Study of the Changing Conditions of Production of Village Blacksmiths in Tanzania

Development Research Series No. 1, Aalborg University Press und Scandinavian Institute of African Studies, 1980, 214 S.

„,Technologietransfer“, ,,Angepaßte Technologie“ sind Stichworte, die seit den 70er Jahren auf internationalen Konferenzen, aber auch in nationalen Entwicklungsplänen zunehmend als Antwort auf die Probleme der Entwicklungsländer genannt werden. Für diese Diskussion um die ,,Wahl“ der angemessensten Technologie besonders in Hinblick auf ihren Nutzen und die Durchsetzbarkeit unter Bedingungen der Weltmarktintegration leistet Jens Müller ausgehend von marxistischen Kategorien einen wichtigen Beitrag.

Müller, der nach eigenem Bekunden für Praktiker in Entwicklungsländern schreibt, versucht zunächst den Begriff Technologie in Beziehung zu Produktionsverhältnissen und Produktivkraftentwicklung zu setzen und für den weiteren Fortgang der Analyse zu operationalisieren. Der Versuch, den hohen Abstraktionsgrad dieser Kategorien für die Praxis handhabbar zu machen, gelingt nur ansatzweise und mündet in der etwas allgemeinen - wenngleich wichtigen - Feststellung, daß Technologie nicht losgelöst von den gesellschaftlichen Rahmenbedingungen betrachtet werden dürfe, mithin keine unabhängige Variable darstelle, die beliebig gewählt und angewendet werden könne. Die eigentliche Stärke der Studie von Müller, der längere Zeit als Entwicklungsberater in Tanzania tätig war, liegt in der empirischen Aufnahme der Produktionsbedingungen der Dorfschmiede. Diese Schmiede könnte in einem so wenig industrialisierten Land wie Tanzania mit seiner einfachen Technologie den die landwirtschaftliche Produktion hemmenden Mangel an Hacken (dem wichtigsten Arbeitsgerät der Bauern) und anderen Werkzeugen beseitigen helfen. Eine einfache Hütte, zu- 
meist selbstgefertigte Werkzeuge wie Hammer, Steinamboß und Blasebalg sowie etwas Eisenschrott und Holzkohle genügen zur Herstellung von Hacken, Sicheln u. a., die zudem genau auf die bestehenden landwirtschaftlichen Arbeitsprozesse und regional unterschiedliche Bodenbeschaffenheiten abgestimmt sind, da ein großer Teil der Schmiede selbst als landwirtschaftlicher Produzent tätig ist oder zumindest um die konkreten Arbeitsbedingungen der Bauern weiß. Als hochqualifizierte Metallfacharbeiter könnten die Schmiede den Grundstock für eine eigenständige technologische Entwicklung für landwirtschaftliche Produktionsmittel bilden, wenn ihr Hauptproblem - die ausreichende Versorgung mit Eisenschrott und Holzkohle - gelöst werden könnte. Doch obwohl die Schmiede selbst unter administrativen Repressionen während der Kolonialzeit ihre Produktion aufrechterhalten konnten und obwohl die Schmiede unter den derzeit ungünstigen Bedingungen 1/5 der gesamten Produktion an Hacken herstellen, droht diese Kapazität nun liquidiert zu werden. Das liegt laut Müller vor allem daran, daß die ,,Staatsklasse“ des im eigenen Selbstverständnis sozialistischen Tanzania unter dem Druck von Weltmarkt und Weltbank, aber auch aus dem Interesse an Mehrwertabschöpfung vor allem exportorientierte Produktionszweige fördert und mit einer entsprechenden Infrastruktur ausstattet. In diesem Rahmen erscheint Müller eine von ihm geforderte indirekte Förderung der Schmiede durch an ihren Bedürfnissen ausgerichtete Infrastrukturmaßnahmen, ,sozio-politisch" nicht machbar, zumal die ausführenden Entwicklungsbürokratien weit mehr an sozialer Kontrolle und der Verbreitung „,moderner" Techniken durch von außen aufgepfropfte ,,Angepaßte Technologie“ interessiert seien als an einer unabhängigen und eigenständigen technologischen Weiterentwicklung.

„Angepaßte Technologie“ entlarvt sich - so Müller - in der Praxis als Ideologie zur Stabilisierung des Status quo.

Peter Schmitz

\section{TeKLewold Gebrehana}

\section{Duty to Negotiate. An Element of International Law}

Svenska Institutet för Internationell Rätt, Uppsala, 1978, XII, 159 S.

Den Leser dieser in Schweden geschriebenen schmalen Dissertation (118 Seiten Text) erwartet anderes, als der Titel vermuten läßt. Es geht Gebrehana nicht etwa darum, ausgehend von bi- und multilateral vertraglich vereinbarten Verhandlungspflichten zwischen Staaten zu fragen, ob (und gegebenenfalls in welchen Bereichen) sich solche Pflichten schon gewohnheitsrechtlich durchgesetzt haben, was zweifellos eine interessante Frage wäre. Der Autor gibt statt dessen eine weit ausholende Skizze, in der er darstellt, wie er und andere die heutige Völkerrechtsordnung sehen. Seine Sichtweise nun unterscheidet sich erheblich von der üblichen. In Kapiteln über ,,The Present State of International Law" und ,,The Sources of Contemporary International Law " versucht Gebrehana zu begründen, daß die gesamte bisherige Rechtsquellenlehre überholt, statt dessen ,,general treaties“ (wie die Satzung der Vereinten Nationen) und ,guiding principles" maßgebend seien. Mit letzteren meint der Autor das Selbstbestimmungsprinzip, die Grundsätze der friedlichen Koexistenz und die ,Duty to Negotiate", die dem Werk den Titel gegeben hat. Der Gedanke, auf den die Ablehnung insbesondere des Völkergewohnheitsrechts gestützt wird, die axiomatische Bedeutung des Konsenses für die internationale Rechtsordnung, wird bei diesem Rückgriff auf naturrechtsähnliche, vage Prinzipien außer acht gelassen.

Verständnis und Lesbarkeit der Arbeit werden erschwert durch eine unübersichtliche Gedankenfolge, zahllose Direktzitate, die of t wahllos aneinandergereiht scheinen, und schließ- 